\title{
Brief Analysis on the Development History of Nanping Pipa Playing and Singing
}

\author{
Juanjuan Cheng \\ College of Music, China West Normal University, Nanchong, 637009, China
}

Keywords: Nanping Pipa, Development history of playing and singing, Brief analysis.

\begin{abstract}
Nanping Pipa as the important component of national level intangible cultural heritage directory represents strong national feature in Northwestern Sichuan plateau. With long history, Nanping Pipa was well received by local people in Northwestern Nanping after introduced from Northwestern area. This article has discussed the origin and expression based on the development history of Nanping Pipa playing and singing, and then introduced some elements of Nanping Pipa playing and singing, and at last given an outlook of the development prospect and protection of the art in nowadays society.
\end{abstract}

\section{Introduction}

Nanping Pipa as a self-play a self-sing folk art in Nanping County is also known as Nanping ditty widely sang among people in Jiuzhaiigouu county of Northwestern plateau. Nanping Pipa known by us now developed fastest in Qing dynasty and spread to Shanxi, Gansu and current area. On September 27th 2015, Nanping Pipa awarded Guinness most large-scale Pipa concert with its clear music quality and profound cultural connotation. In addition, Nanping Pipa playing and singing is also national intangible cultural heritage. The author tries to introduce and elaborate the development history of Nanping Pipa playing and singing to get people to further know Nanping Pipa playing and singing, the traditional folk art, understand the development origin, expression form and features of it, so as to stimulate people's awareness of protecting it.

\section{Development origin and expression form of Nanping Pipa playing and singing}

\subsection{Origin}

Nanping Pipa also called Nanping ditty or Nanping minor is a self-play and self-sing art form. According to historic records and description, it was introduced by immigrants of Shanxi and Gansu in Emperor Yongzheng and Jiaqing of Qing dynasty. With the change of time and environment, it starts integrating with north and south culture into an entirety and forms into Nanping ditty with Northwestern Sichuan features.

With long history, Nanping Pipa has rich cultural connotation. Usually it is performed by players in joyful or festivals as a collective entertainment activity with strong local flavor. As an excellent traditional folk art in Jiuzhaigou, Sichuan, its biggest feature is to play and sing beautiful melody in local dialect with simple but characteristic lyrics, most of which from folk oral literature.

About Jiuzhaigou of Sichuan, there is a sentence spreading among people, Jiuzhaigou is the world and sea of playing and singing. The reason can be explained form the historical origin of Nanping Pipa playing and singing. Long ago when people lived on planting corps and started playing Pipa and singing together with friends or families after a full day's hard work so as to relieve fatigue and loneness. Especially in wedding ceremonies or festivals, local folks get together to celebrate by playing Pipa and singing. The scene was extremely boisterous. Under the cultivation of simple folk custom, Pipa playing and singing is developing with more national features and folk styles. 


\subsection{Development of Nanping Pipa}

According to the elaboration of the origin of Nanping Pipa, we have a general understanding that it is Nanping ditty widely sang among people in Jiuzhaiigouu county of Northwestern plateau which is located in remote mountainous area with inconvenient transport so as to impede the transmission and development of Nanping Pipa. Before the foundation of New China, it was a self-play and self-sing entertainment activity among local people with great simplicity and originality.

At first, melodies of Nanping Pipa playing and singing was created based on original Qupai, added with some simple fudiao organizers and new words and performed by female chorus. In addition, the accompaniment has been changed with Yangqin and Erhu introduced based on Pipa and tea dish. But it is still performed in dialect.

Then there are some new changed in original Qupai with phoneme of traditional sanxian pipa adjusted and standing singing and figure techniques newly added. In this way, the overall effect of Nanping Pipa playing and singing is more colorful.

At last, up to now, there are several kinds of Pipa playing and singing songs created with original Qupai as basic materials. We need to pay attention that previous qipin sanxian Pipa is changed to sipin Pipa and added to Yangqin, Zhong ruan, and Guzheng, etc. performed in Chengdu dialect.

\subsection{Expression content of Nanping Pipa}

The expression content of Nanping Pipa is diversified as it is closely related to the genre of Nanping Pipa playing and singing. Thus this paper can elaborate its expression contents based on the type of Nanping Pipa playing and singing.

Firstly, historical traditional type. This genre is largest in number with the content of praising heroes and courage. Some are historical stories and legends such as well-known "MengJiangNu's bitter weeping", "Boya breaks the instrument", and "Luoyang Bridge" and other long narrative folk songs.

Second is about love romance. This genre is to express love between men and women, and vicissitudes of life. This kind of playing and singing is with strong mark of feudalism such as "Embroider Wallet", "Love song", etc.

Thirdly, labor life genre is to reflect people's production and life by virtue of Pipa playing and singing. Well-known works include "Returning home" and "Pick grapes", etc.

In a word, these famous songs are performed with unique Nanping Pipa with finger cymbals and porcelain dishes used as percussion music.

\subsection{Spreading range of Nanping Pipa playing and singing}

As mentioned above, Nanping Pipa playing and singing is Nanping ditty widely sang among people in Jiuzhaiigouu county of Northwestern plateau which is located in remote mountainous area. Nanping refers to Jiuzhaigou. Although there are a lot of legends about the place, scientific exploration should have real evidence and clues. Many cultural relic earthed are from new period. Due to the special location, surrounded by mountains, it is the best place for concealing. So this is divided as external area to defend enemy's invasion, which results in the subsequent migration of troops and pollution to the place living with local people. The large-scale migration leads to local economic and cultural flow.

\subsection{Features and categories of Nanping Pipa playing and singing}

Nanping Pipa playing and singing is also called Nanping melody, basically performed by playing and singing, accompanies by porcelain dishes or dumbbell. In addition, the performance is various, usually by solo or chorus, sanxian Pipa mainly used for accompaniment. Especially in rural or towns, it is very popular as the major activity for labor people at leisure time and indispensable component of mountain people. Furthermore, there are usually two melodies: Huadiao and Beigongdiao. The former is simple in tonality and content such as the well-known "Flower picking", but the latter is complex comparatively, such as well-known "selected scenes" and "Master picks up his robe".

As for the category of Pipa playing and singing can be divided into two types: melodies performed 
in showing festive lantern in Spring Festival, also called "flower melody", such as well-known "My beloved brother", and "Jasmine flower", and "Ten words". Another kind is for festival travelling and relaxation, also called "Beigong melody", such as well-known "Boya breaks his instrument", "Hang red lantern" and "Fu Lu Shou Xi".

\section{Overview of Nanping Pipa playing and singing elements}

\subsection{Playing technique}

Nanping Pipa playing and singing methods are similar with when first introduced into China, holding by hands sideways and playing with fingers. It can be summarized into three words: play, pick and whisk.

\subsection{Playing form}

Nanping Pipa belongs to accompaniment of musical instruments in terms of function, so its playing form changes according to the requirement of song singing by solo and chorus. Also it can be played by antiphonal singing and chorus in harmony with the leading singer. In performance gesture, it is performed by seated singing or standing singing but not usual. It can be divided into single -singing or multiple singing. The former refers to one person responsible for playing and singing, another for accompaniment. The latter refers to one or more people responsible for playing and multiple people responsible for singing, the rest responsible for accompaniment by different instruments.

\subsection{Theme}

\subsubsection{Melody style}

Melody style of Nanping Pipa playing and singing is influenced by quzixi singing technique in Shanxi and Gansu, also called mei and hu county theatre, not only so, the performance style is directly related to the melody style of "Huaer" in Gansu and Qinghai area. Integrated with other melodies and singing technique, Nanping Pipa has formed its own melody styles. But there are two distinctive features: firstly, there is an invariable four-section intro in the whole musical structure, which makes Nanping Pipa playing and singing more recognizable so as to establish local people's unique feeling for it. Secondly, the element of the ending of every melody is basically the same. Thus Nanping Pipa playing and singing has integrated the dialect of Shanxi, Gansu, and Northwestern Sichuan, Tibet, and also owns the playing and singing ditty with unique characteristic and connotation of Jiuzhaigou.

\subsubsection{Structural features of lyrics}

According to relevant investigation, and literature records, in the content of 80 melodies well organized, most lyrics of the songs use rhyme structure and few have the structure of rhyme and dispersion. The content expression style shows free, resounding and euphemistical style. The detailed expression contents are about love stories, historic legends and production and life.

\section{Development prospect and protection of Nanping Pipa playing and singing art}

With unique geographic position and terrain landform, Jiuzhaigou has awarded as national natural reserve and national key tourist attraction, world natural heritage, and other honors. As China's famous tourist spot, it has attracted visitors from home and overseas. Nanping Pipa playing and singing as the traditional and characteristic folk art with long history has been incorporated in world intangible cultural heritage directory. We need to get more people to know and understand the folk art. With more visitors in Jiuzhaigou, the government should strengthen the publicity and promotion of Nanping Pipa playing and singing art while developing tourism. For example, present it as important performance in front of visitors in holidays or theme activities. Meanwhile, government should take 
other measures such as launching Nanping Pipa into campus activities, cultivating Pipa professional teachers, and energetically bringing in professional music talents, organizing Pipa playing and singing course, so that the development history and cultural connotation of Pipa playing and singing can be known by more people. In addition, the government and relevant departments should give financial or spiritual support for the existing producers of Nanping Pipa and prevent it from being lost. With the guarantee and support of relevant policies and measures, the author believes that Nanping Pipa playing and singing, the excellent traditional folk art will have broad development prospect.

\section{Conclusion}

To sum up, Nanping Pipa playing and singing as traditional folk art form in Jiuzhaigou County gathers blood and wisdom of Chinese people from generation to generation, as well as the real treasure. With the change of time, it should timely adapt to social development trend. From the spreading area or art genre, or playing elements have changed accordingly. This paper has briefly summarized the development history of Pipa playing and singing so as to help reader further understand the folk art essence and also present the century-old art in front of people's eyes. China pays great attention to intangible cultural heritage protection, Naping Pipa as an important part of national level intangible cultural heritage has promising development prospect.

\section{Acknowledgement}

China West Normal University Excellent Talent Plan "Exploration and studies on Nanping Pipa singing and playing art" (Project number: 17YC540), China West Normal University innovation team "Studies on Bashu folk musical culture and database construction" (Project number: CXTD2016-8).

\section{References}

[1] Wang Xuemei. Sumamry of comparison between Nanping Pipa and modern general Pipa, Yellow River of the Song, 2017-01-06.

[2] Yao Xue, Evolution and application of Pipa right-hand technique since Ming and Qing Dynasty, Wuhan Conservatory Of Music, 2017-03-31.

[3] Shui Yuan, Shilin, New generation of Pipa singing and playing, Nanguo Hongdou, 2014-01-25.

[4] Cui Xiaojun. Chinese traditional culture and Pipa art, Popular Literature, 2017-02-15.

[5] Zhou Qingbao. Trace the source of Pipa, Journal of Sichuan Conservatory of Music, 1985-10-01. 